\title{
Technology for the Formation of Functional Coatings Based on Glass Microspheres
}

\author{
Alexey Kuchugurov ${ }^{1}$, Nikolay Shardakov ${ }^{1, *}$, Inna Kovyazina ${ }^{2}$, and Nadezhda Lukoyanova ${ }^{1}$ \\ ${ }^{1}$ Ural Federal University, Glass Technology Department, 620002, 28 Mira str., Yekaterinburg, Russia \\ ${ }^{2}$ Ural Federal University, Department of Foreign Languages and Educational Technologies, 620026, \\ 48a Kuibysheva Street, Yekaterinburg, Russia
}

\begin{abstract}
The technology for the formation of functional coatings from hollow glass microspheres by the self-organization method from aqueousalcoholic suspensions is proposed. A monolayer with the closest (hexagonal) packing is shown to be formed on a solid (glass) substrate. The criterion for the ordering of a monolayer is proposed. The assessment of the influence of various technological factors on the monolayer formation rate and the degree of its ordering is given.
\end{abstract}

Layers of glass microspheres are considered to be promising materials as reflective [1] and heat-insulating $[2,3]$ coatings, devices for concentrating of light energy in nanolithography and solar energy [4]. Light focused by a microsphere called "photonic stream" can be also applied in optical traps for manipulating nanoobjects, and designing microscopes with a resolution lower than the wavelength. Single microspheres are micro resonators with a very high quality factor [5], which makes them attractive for the development of microlasers.

Currently, microspheres are widely used in the manufacture of reflective coatings which are a multilayer structure including, inter alia, a layer of microspheres of the same diameter and a reflective layer. Microspheres the coating are arranged in a certain order which is achieved by placing them in an underlying layer with a cellular structure.

When developing heat-insulating coatings the requirement for monodispersity of particles and their arrangement ordering is not mandatory. However, the heat-insulating properties of such coatings are emphasized to improve with an increase in the number of contacts between microspheres. The latter can be achieved by collecting microspheres in the closest packages.

Thus, the properties of both reflective and heat-insulating coatings are critically dependent on the degree of ordering of microspheres in these layers. Therefore, the development of a technology for obtaining close-packed layers of microspheres is a rather urgent problem.

The currently proposed technologies for the manufacture of reflective coatings are based on the principle of mechanical placement of microspheres, include a large number of operations, and require complex precision equipment [6]. Microspheres with the diameter of $50-150 \mu \mathrm{m}$ are used in the manufacture of coatings, as a rule. The packing of particles of such sizes into ordered structures presents certain difficulties. They are small for mechanical

\footnotetext{
* Corresponding author: n.t.shardakov@urfu.ru
} 
packing methods, and it is difficult to capture and hold them by mechanical devices. The method of self-organization in dispersion media is also difficult to implement due to the relatively large mass of particles and their low mobility. This conclusion, however, is valid for massive particles - microspheres. If hollow microspheres are used, the mass of which is much less, the phenomenon of self-organization for the formation of an ordered structure becomes, in our opinion, quite possible. Such studies on the formation of ordered structures from hollow glass microspheres by the method of self-organization in a dispersion medium are not provided, though.

The aim of the work was to develop a technology for the formation of a monolayer of hollow glass microspheres with the diameter of $110-125 \mu \mathrm{m}$ on a solid (glass) substrate. In addition, it was necessary to investigate the structure of the resulting packings, formulate criteria for assessing the quality of the obtained ordered structures, and assess the influence of technological factors on the quality of ordered structures.

Microspheres were made of sodium aluminosilicate melt saturated with sulfur oxides. After cooling the melt, pieces of glass were crushed to obtain a powder of a granulometric composition given. Then, the powder was fed into a high-temperature furnace where the particles were melted to become a spherical shape. Simultaneously, as a result of gas evolution, gas cavities were formed inside the particles. Then, at the stage of flotation the hollow microspheres were separated from the microbeads and destroyed fragments of the microspheres. After flotation, the microspheres dried had the bulk density of $\sim 0.2 \mathrm{~g} / \mathrm{cm}^{3}$ and the diameter of $10-150 \mu \mathrm{m}$, the wall thickness was $\sim 1 \mu \mathrm{m}$.

The fraction of 110-125 $\mu \mathrm{m}$ selected on a vibrating sieve was washed with pure ethyl alcohol while additional separation of microbeads and destroyed fragments of microspheres with a density greater than that of ethanol occurred. Fragments and microbeads sank to the bottom of the vessel, and undamaged microspheres floated on its surface. Then, the microspheres collected from the surface were dried and a suspension was prepared on their basis. The dispersion medium for the suspension was a mixture of distilled water and ethanol. The ethanol content was varied from 0 to 100 vol. \% with a step of 5 vol. $\%$.

Drops of the suspension were applied with a syringe to glass substrates $(30 \times 40 \times 2 \mathrm{~mm})$ cut from standard photographic plates; preliminarily the photosensitive layer was washed off with hot water, then the plates were dried and degreased with ethyl alcohol.

To evaporate moisture a substrate with drops of suspension was placed either in a cabinet drier heated to $50^{\circ} \mathrm{C}$ or in a sealed vessel (desiccator) where the humidity was maintained at $63 \%$ or close to $100 \%$ at room temperature. To maintain a humidity of $63 \%$ a saturated solution of sodium nitrate was poured into the desiccator, and distilled water was poured into the desiccator to maintain the humidity close to $100 \%$.

Since the aim of this work was to obtain a monolayer of microspheres with the closest (hexagonal) packing when each microsphere is surrounded by six nearest neighbors, the number of hexagonal cells of microspheres per unit area of the monolayer was proposed to be considered as a criterion of ordering (Fig. 1). To count the cells the image of a monolayer was displayed on a computer screen using an MBS-9 stereoscopic microscope and a video camera and then processed using a graphics program. 


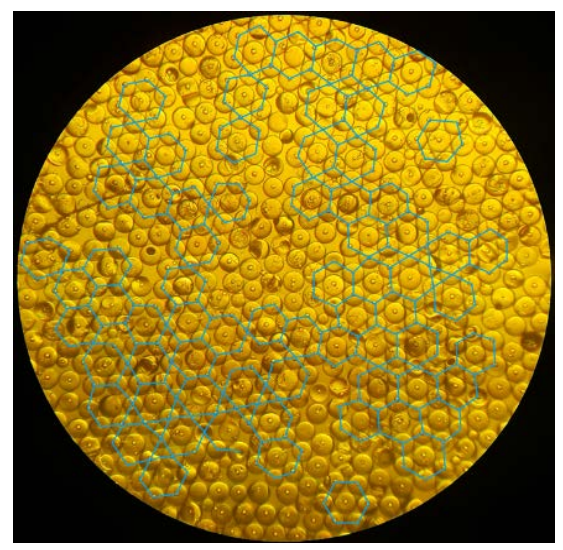

Fig. 1. Isolation of hexagonal cells in a monolayer of microspheres

Preliminary experiments have shown that the best results are obtained when drying occurs at room temperature and high humidity (Table 1). Therefore, further studies were carried out at the humidity of $\sim 100$ vol. $\%$ and the temperature of $(23 \pm 3){ }^{\circ} \mathrm{C}$.

Table 1, Conditions for the formation of a monolayer of microspheres

\begin{tabular}{|c|c|c|c|c|c|c|}
\hline $\begin{array}{c}\text { Formation conditions and } \\
\text { ordering criterion of a } \\
\text { monolayer }\end{array}$ & 1 & 2 & 3 & 4 & 5 & 6 \\
\cline { 2 - 7 } & 0 & 0 & 0 & 1 & 10 & 40 \\
\hline Ethanol content, vol. \% & 63 & 63 & $\sim 100$ & $\sim 100$ & $\sim 100$ & $\sim 100$ \\
\hline Humidity of the atmosphere, $\%$ & 23 & 50 & 23 & 23 & 23 & 23 \\
\hline $\begin{array}{c}\text { Temperature, }{ }^{\circ} \mathrm{C} \\
\text { Number of hexagonal cells on } \\
\text { the substrate surface, } \mathrm{mm}^{-2}\end{array}$ & $54 \pm 11$ & $60 \pm 23$ & $105 \pm 15$ & $97 \pm 11$ & $161 \pm 10$ & $118 \pm 18$ \\
\hline
\end{tabular}

According to the results of the study, the dependence of the microspheres monolayer ordering degree on the concentration of ethanol is nonmonotonic - the maximum number of hexagonal cells is observed on the substrate surface at the concentration of $\sim 13$ vol. \% (Fig. 2).

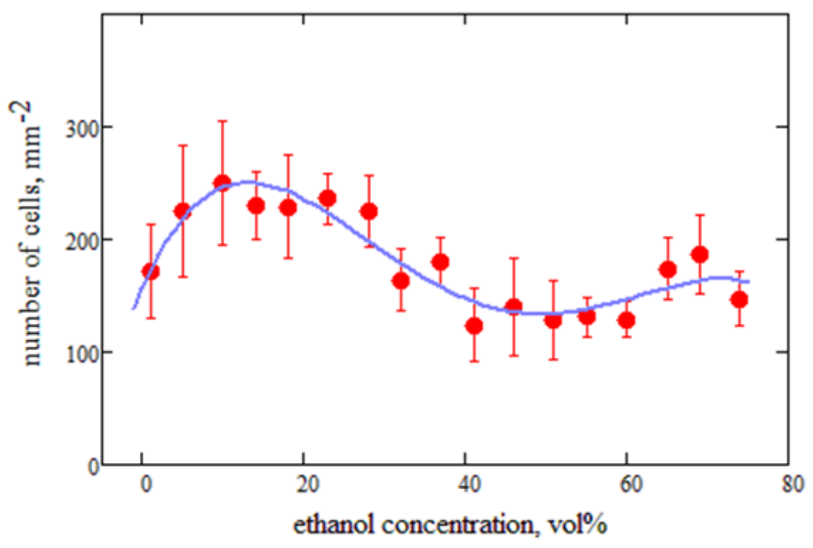

Fig. 2. Dependence of the monolayer ordering on the ethanol concentration 
Three forces act on a microsphere on the surface of a liquid: gravity, Archimedes' force, and capillary (surface) forces. The first two are directed along the normal to the surface and do not affect the movement of particles along the surface. Capillary forces are directed along the surface. Particles under capillary force action can approach to or move away from each other depending on the value of the contact angle. As the angle of glass wetting with an aqueous solution of ethanol is less than $90^{\circ}$, the meniscus between the particles is concave, and the pressure in the liquid under the meniscus is less than atmospheric (Fig. 3).

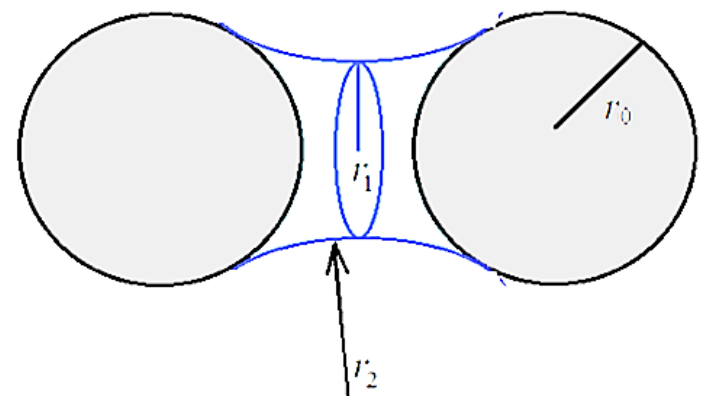

Fig. 3. Meniscus shape of liquid with positive wetting of particles

In this case, the capillary pressure $p_{\sigma}$ (the difference between the atmospheric pressure and the pressure inside the liquid) pushed the particles against each other

$$
p_{\sigma}=\sigma\left(\frac{1}{r_{1}}+\frac{1}{r_{2}}\right) \text {, }
$$

where $r_{1}$ and $r_{2}$ are the main radii of curvature, $\sigma$ is the surface tension of the liquid.

With a vanishingly small amount of liquid between the particles (their maximum approach) the force pulling together the particles is determined by the size of the particles $\left(r_{0}\right)$ and the value of the surface tension

$$
F=2 \pi r_{0} \sigma .
$$

The Brownian motion prevents the particles from approaching: the more intense is, the higher the temperature is. Therefore, the temperature during the formation of a monolayer must not be too high. However, too low temperatures are also undesirable, since the final position characterized by the minimum energy must be found by the particle by repeatedly enumerating the positions. For the same reason, the rate of evaporation of liquid from the suspension must not be too high that is achieved by creating increased humidity.

Thus, the formation of an ordered monolayer of hollow glass microspheres of the given diameter by the self-organization method from a suspension is most significantly influenced by three factors: the surface tension of the dispersion medium, temperature, and humidity. These factors have the opposite effect on the monolayer formation rate: an increase in surface tension increases it, but an increase in temperature and humidity decreases it. To obtain a monolayer with maximum ordering an optimal combination of these factors should be performed.

\section{References}

1. Yu.M.. Belyakov, E.V. Zverev, E.R. Galimov, Yu.A. Pryakhin, E.E. Tukbaev, Optical model of reflectors with microspherical reflectors, Problems of power engineering J., 5-6, pp. 129-133 (2007) 
2. V.M. Kiselev, A.A. Burchinov, V.M. Volynkin, S.K. Evstropiev, A.V. Matveentsev, Light-absorbing composite coatings based on hollow oxide microspheres and lead sulfide, Optical J., v. 82(12), pp. 60-64 (2015)

3. A.I. Efimova, V.B. Zaitsev, Optical properties of polydisperse ensembles of microglass spheres in the infrared spectral range, VMU. PHYSICS. ASTRONOMY J., v 3(2), pp. 83-88 (2018).

4. I.V. Minin, O.V. Minin, Photonic streams in science and technology, Vestnik of SSUGT J., v 22(2), pp. 212-234 (2017)

5. M.L. Gorodetsky, Fundamentals of the theory of optical microcavities (Moscow: Faculty of Physics, Moscow State University 2010)

6. T.N. Vagizov, E.R. Galimov, Yu.A. Pryakhin, Technologies for obtaining reflective coatings with specified properties, Vestnik KAI J., 3, pp. 49-53 (2017) 